Document downloaded from:

http://hdl.handle.net/10251/94492

This paper must be cited as:

Payri, R.; Gimeno, J.; Marti-Aldaravi, P.; Manin, J. (2012). Fuel concentration in isothermal Diesel sprays through structured planar laser imaging measurements. International Journal of Heat and Fluid Flow. 34:98-106. doi:10.1016/j.ijheatfluidflow.2011.12.007

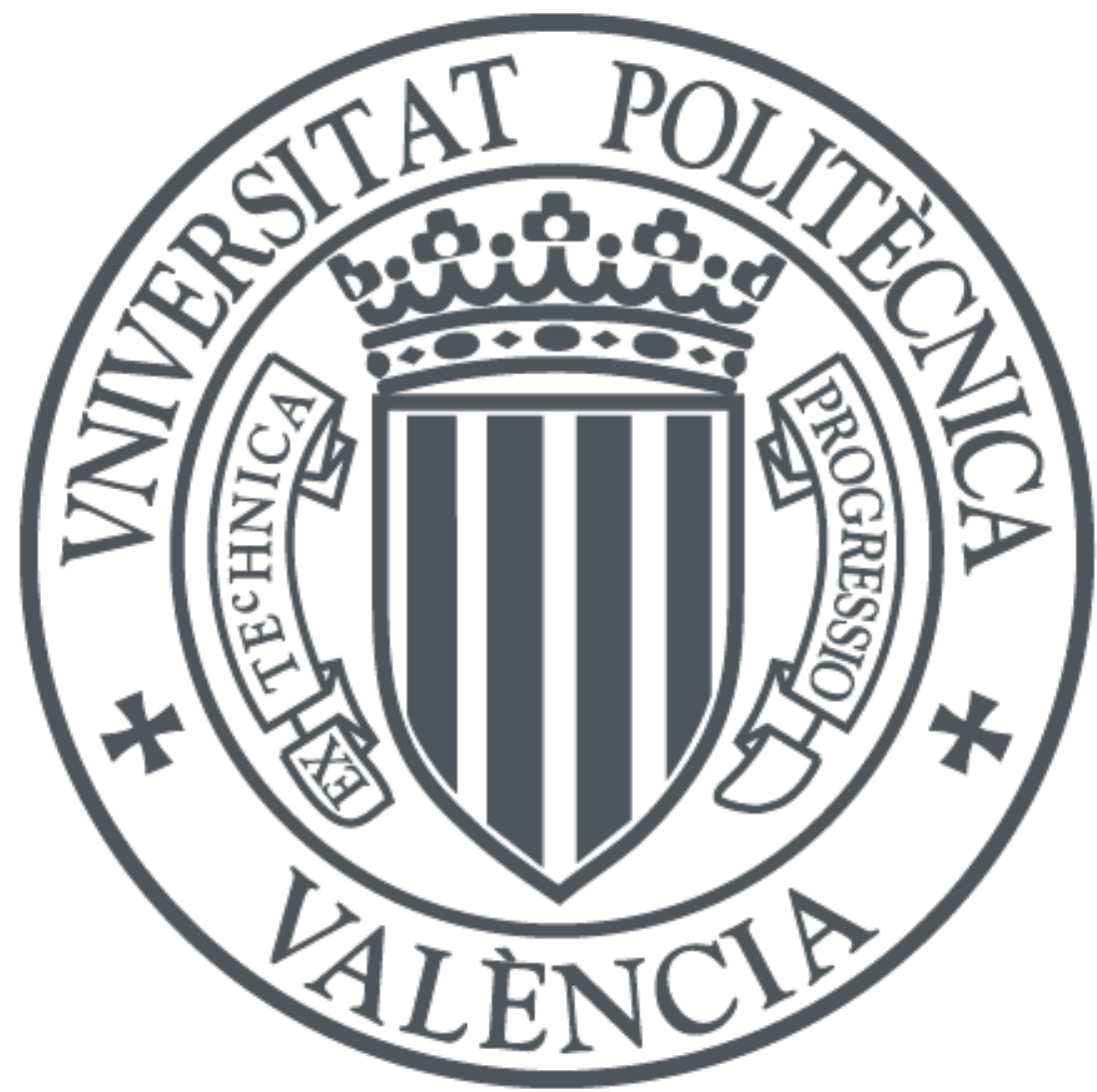

The final publication is available at

http://doi.org/10.1016/j.ijheatfluidflow.2011.12.007

Copyright ELSEVIER SCIENCE INC

Additional Information 


\title{
Fuel concentration in isothermal Diesel sprays through structured planar laser imaging measurements
}

\author{
R. Payri, J. Gimeno, P. Martí-Aldaraví, J. Manin \\ CMT Motores T'ermicos, Universidad Polit'ecnica de Valencia, Camino de Vera s/n, 46022 Valencia, \\ Spain
}

\begin{abstract}
The mixing of isothermal liquid sprays in engine-like conditions has been investigated by applying the Structured Planar laser Imaging technique to remove multiple light scattering. The intensity of the illumination plane has been recovered by removing multiply scattered light and mapping the spray three-dimensionally via discrete tomography. Based on the extinction of light within the illumination plane, the number density has been extracted. Coupled with 2-D maps of droplet diameters obtained through LIF/Mie ratio, the number density allowed to calculate the fuel concentration in the sprays. The mixture fraction of DI Diesel sprays injected into an inert environment held at room temperature has been evaluated and compared to a validated model based on gas-jet theory. The experimental results showed good agreement with the predictions when assuming a Gaussian radial distribution and tuning the Schmidt number. A Schmidt number below the unity demonstrated to be the best fit to the results. In addition, the Gaussian distribution showed to be a good assumption based on the closeness to the experiments, although some differences in shape have been noticed. For different testing conditions, whilst the influence of ambient density on mixing was expected, the effect of injection pressure brought up additional information concerning the global mixing of liquid sprays.
\end{abstract}

Keywords:

Diesel sprays, Fuel concentration, Laser diagnostics, Fluorescence (LIF).

\section{Introduction}

Achieving a homogeneous air-fuel mixing is the key parameter concerning combustion efficiency in internal combustion engines [1]. In direct injection Diesel engine, the injection law mainly drives the combustion process and therefore, the global mixing is directly related to the development of sprays $[2,3,4]$. At the same time, analyzing sprays out of a multi-hole nozzle in an engine is really challenging and for that reason, researchers often have recourse to specific installations. Injecting sprays in an isothermal environment is simple and their similarity to gaseous jets make such study a powerful tool to analyze mixing processes $[5,6]$.

For more than two decades now, the laser-based technique are widely used to analyze the specificity and complexity of sprays. The planar imaging offers several advantages, the main one being to generally allow quick and global analysis of the entire spray. Planar laser imaging relies on the fact that a section of the spray can be cut by a thin laser-sheet. The laser radiation propagates through the droplets and transmits the light according to an elastic scattering process governed by the Lorenz-Mie theory formulated more than a century ago. These independent for-

Email address: rpayri@mot.upv.es (R. Payri) mulations have been adapted to modern physic and have been extensively applied in engine researches mainly to study the liquid part of the spray.

The Planar Laser-Induced Fluorescence (PLIF) is an extension of the planar laser imaging technique. Used either for gases or liquids, it assumes that the quantity (or mass) of the substance under study is proportional to the fluorescent emission intensity; since this is true, the concentration of the substance can be obtained.

The relative Sauter Mean Diameter can also be calculated by dividing the two signals obtained, i.e. fluorescence and elastic Mie scattering (LIF/Mie ratio). Proposed in 1993 by Yeh et al. [7] this 2-D droplet sizing technique assumes surface dependency with droplet diameter for the elastically scattered light signal and volume dependency for the fluorescent emission. Since then, many researchers concentrated their efforts on this field and spray analysis using the LIF/Mie ratio, commonly called PDS (Planar Droplet Sizing), became more and more important.

There are, however, some problems when the objective is to study dense optical media like modern Diesel sprays with planar laser imaging mainly due to multiple light scattering [8]. With the improvements made in the field of experimental techniques, some solution became available to go deeper in such dense ensemble of droplets. Ballistic imaging [9] or X-Ray tomography [10] demonstrated 
their ability to perform measurements in dense regions of liquid sprays. However, the requirements in terms of equipment make these experimental solutions available to a few researchers only. A novel technique (SLIPI) based on structured illumination has been developed to study liquid sprays and showed great potential to remove multiply scattered photons [11]. The limitations of the technique have been identified to be the requirement of at least three phase-shifted images (i.e. three phases) and the dynamic range of the imaging system.

The objective of the present work is to developed an alternative to the SLIPI technique with only two phases called SPLI-2P and apply it to the Mie and LIF signals of an isothermal Diesel spray. This paper is divided into three primary sections: first, the physical background, then, the calibration of the experiments and finally the results as well as their validation thanks to a jet model [12]. The physical background quickly presents the theory concerning the propagation of light in dense media either for Mie or LIF processes. The principle of the SPLI-2P technique to measure the mixture fraction in non-evaporative Diesel sprays is also presented in this first section together with the advantages and limitations. The fluorescence properties of the fuel used are described in the calibration section with an theoretical evaluation of the parameters defining the PDS technique. The last main section presents the results and compare them to a model based upon gasjet theory. The conclusions concerning the measurement of the fuel mass fraction in a spray injected under nonevaporative conditions are finally drawn.

\section{Physical background}

The mathematical representation of the interaction between electromagnetic waves and particles, best known as the Mie theory, stands for the absolute reference to study the diffusion of light by particles. It is possible, from a theoretical approach, to extract the droplet size and number density of an ensemble of particles by collecting the fluorescent and scattered signals. From a practical point of view, the initial signal coming from the illuminated particles is affected by multiple scattering the collected information is the result of both single and multiply scattered photons. By illuminating the sample with a structured signal, the multiply scattered light can be separated and the singly scattered signal only can be extracted.

\subsection{Transmission of light in turbid media}

The propagation of electromagnetic waves into a collection of particles is subject to attenuation and multiple scattering phenomena. Those processes may induce errors when measurements of droplet size and concentration are performed and depend on the scattering regime, droplet size distribution and optical depth $\tau$ principally [13]. Multiple scattering is responsible of changes in the trajectories of the photons which interact with the particles causing losses in the original singly scattered signal emitted by the droplets. This deviation of the photon mainly depends on particle size and refractive indices and can be quantified by the measure of the optical depth.

Another process can affect the light signal which propagates in the medium: the absorption. This process depends upon the physicochemical properties of the substance under study and is wavelength dependent. When a fluorescent dye is added to the particles or droplets, the signal emitted is directly proportional to the absorption of this substance [14]. The combined effect of these two processes (scattering and absorption) is called extinction and describes the attenuation of light traveling into a turbid media. The Beer-Lambert law is commonly used to express the extinction of light traveling into an optically thick homogeneous medium with constant extinction along the path such that:

$$
I_{t}=I_{0} \exp \left(-\mu_{e} L_{o p t}\right)
$$

where $I_{0}$ and $I_{t}$ are the intensities of the incident radiation and the transmitted light exiting the sample in the original direction, $L_{\text {opt }}$ is the pathlength of the sample under study and $\mu_{e}$ is the extinction coefficient which can be expressed as the sum of the scattering coefficient and the absorption coefficient:

$$
\mu_{e}=\mu_{s}+\mu_{a}
$$

Each of these coefficients is proportional to the number density $N$ (number of particles per unit volume) and to the respective extinction, scattering and absorption crosssection $\sigma_{e}, \sigma_{s}$ and $\sigma_{a}$ as:

$$
\mu_{e}=N \sigma_{e}, \mu_{s}=N \sigma_{s} \text { and } \mu_{a}=N \sigma_{a}
$$

The optical depth $\tau$ can be defined from the two previous equations such that:

$$
\tau=\mu_{e} L_{o p t}=N \sigma_{e} L_{o p t}
$$

The optical depth measures the extinction of light through a turbid media, as shown in equation 4, it corresponds to the product of the number density, the cross-section and the distance within the sample. The extinction crosssection $\sigma_{e}$ is the result of the scattering and absorption cross-sections such as: $\sigma_{e}=\sigma_{s}+\sigma_{a}$ (see analogy with eq. 2).

For polydisperse particles much bigger than the wavelength, an equivalent extinction cross-section $\sigma_{e}$ can be introduced which is expressed as follows:

$$
\sigma_{e}=\frac{1}{N} \sum_{i}^{N} \frac{\pi d_{i}^{2}}{4} Q_{e i}
$$

The extinction efficiency $Q_{e}$ can be considered constant for particles much bigger than the wavelength $(\lambda=532$ $\mathrm{nm})$ and from this last expression, the intensity of light 
traveling into a medium of polydisperse droplet can be written as:

$$
I_{t}=I_{0} \exp \left(-\sum_{i}^{N} \frac{\pi d_{i}^{2}}{4} Q_{e} L_{o p t}\right)
$$

By derivation of the Beer-Lambert law presented in equation 1, the number density of the particles within the sample of interest can be extracted:

$$
N=\ln \frac{I_{0}}{I_{t}} \frac{1}{L_{o p t} \sigma_{e}}
$$

The particle number density is then only function of the incident and transmitted intensities, the optical pathlength and the extinction cross-section (see eq.5).

In the case of mass fraction measurement through LIF technique, the intensity of light exiting an optically thick medium has been attenuated all along its path by both scattering and absorption. However, the multiple scattering phenomenon contorts the signals emitted by the original beam traveling through the cloud of particles. As the photons are deviated, the information of the incident beam is lost during the way to the detection system and some corrections are needed to recover the original information of the central part of the beam.

\subsection{Structured Planar Laser Imaging}

The main idea of SLIPI is to remove the multiple scattering that a planar electromagnetic wave suffers in a medium containing scatterers. This technique relies on spatial modulation of the input light to suppress the unwanted interferences caused by multiple scattering. Structured light has been used in fluorescence microscopy for a decade now and it demonstrated to improve image quality and back scattering detection [15]. A couple of years ago, Breuninger et al. [16] applied a structured illumination together with the so called Planar Laser Imaging technique and used it to study the fluorescence spectroscopy of a biological sample.

A similar technique has been developed to suppress multiple Mie scattering in dense two-phase flows relying on the fact that the radiation remains structured if it suffers one scattering event only. In other words, the photons that have experienced several scattering events within the medium will lose the modulation information contained in the excitation light, while singly scattered photons will not. The signal of the electromagnetic plane wave going into the sample results in a normalized amplitude fringe pattern $a_{m}(x, y)$ of the functional form:

$$
a_{m}(x, y)=1+k_{m o d} \cos \left(2 \pi \nu y+\phi_{0}\right)
$$

Where $k_{\text {mod }}$ represents the modulation depth, $\nu$ the spatial frequency, $\phi_{0}$ is an arbitrary spatial phase while $\mathrm{x}$ and $\mathrm{y}$ are the two dimension coordinates. This function of illumination would then provide a two-dimensional image of intensity $I_{R}(x, y)$ such as [16]:

$$
I_{R}(x, y)=I_{C}+I_{S} \cos \left(2 \pi \nu y+\phi_{0}\right)
$$

In this last equation, a decomposition can be done, $I_{C}$ is one part of the image in which both single and multiple scattering are present. This picture $\left(I_{C}\right)$ describes how the captured image would be if there were no spatial modulation of the incident light. The second part of the image $\left(I_{S}\right)$ contains mainly singly scattered photons and represents the structured illuminated image. The cosine term is the superimpose fringe pattern of the excitation light and must be removed to get the clear structured image $\left(I_{S}\right)$. By changing the phase of the modulation, the illumination will be spatially shifted while the multiple scattering will not be affected.

Recent developments of the structured illumination technique use at least 3 phases (images) to recover the spray and suppress multiple scattering by moving the phase a third of a period leading to relative spatial phases of $\phi_{0}=$ $0,2 \pi / 3$ and $4 \pi / 3$. The choice of 3 phases makes sense as the mathematical process of suppression is straightforward (pair-wise difference) and the image is reconstructed directly.

On the other hand, accounting for the equipment available in laboratories working on sprays all over the world (e.g. double-pulsed laser, double-shutter camera), a twophase solution seems more appropriate. The two phases in this case are shifted by half a period one to each other (relative phases would be: 0 and $\pi$ ). Finally, the following equation represents the resulting signal of the Structured Planar Laser Imaging (SPLI-2P) technique:

$$
I_{S}=\sqrt{(I(0)-I(\pi))^{2}}
$$

The resulting image is then obtained by recreating the upper envelope of the signal as the lower envelope should go to absolute 0 . In the same time, the signal of the nonstructured image referred to as conventional in this work would be:

$$
I_{C}=\frac{I(0)+I(\pi)}{2}
$$

The fact that both signals are contained in the set of images previously acquired makes all kind of comparison easy. At first, every individual phase-image shows a global blurring effect due to the high optical depth of the dense medium probed and therefore a large probability of multiply scattered photons. On the other hand, the singly scattered photons carry the structural information of the phase shift and remain on the post-processed resulting image. This process shown in figure 1 operates as a directional filter and extracts most of the singly scattered photons, removing the multiply scattered ones at the same time, leading to significantly improving the contrast of the original signal. 

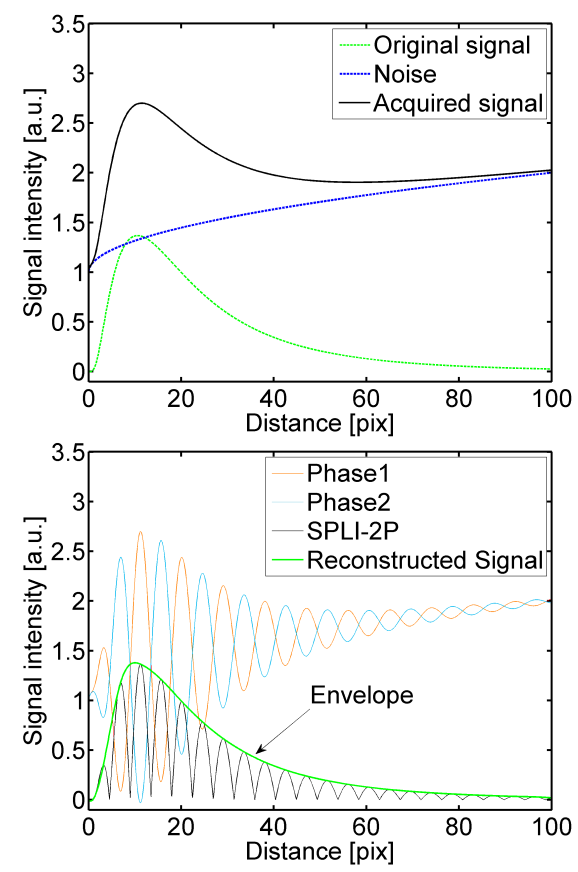

Figure 1: Schematic of the conventional and Structured Planar Laser Imaging (SPLI-2P) methodologies. The signals are shifted by half a period.

In addition to less equipment requirement, the twophase process showed not to be as much affected as the original SLIPI by a mis-shifting of the phases and is therefore more robust at removing multiple scattering.

\section{Setup and calibration of the experiments}

The sprays are generated by a purposely-designed injection system capable of holding the pressure constant with control and accuracy. The nozzle used for this study is a single-hole nozzle, solenoid-actuated used in the ECN Working Group [17] with a diameter of $0.09 \mathrm{~mm}$ and $k$ factor $=1.5$. An additional equipment has been designed to control the temperature of the injector as this may affect the fluid and the hydraulic properties of the injection system itself.

\subsection{Optically accessible cold spray facility}

The visualization test rig is a closed-loop installation designed in order to simulate the air density in the combustion chamber of an engine prior to injection. The advantage of such a test rig is the optical access that allows better visualization of the spray than in a real engine where the moving elements make any kind of visual measurement highly challenging.

The rig is filled with sulfur hexa-fluoride $\left(S F_{6}\right)$, which is an inert gas and has similar characteristics like air in terms of viscosity and optical properties [18]. A constant gas stream flows in the closed-loop at a velocity lower than $3 \mathrm{~m} / \mathrm{s}$ to ensure complete scavenging of the testing volume between two injection events. The temperature of the gas

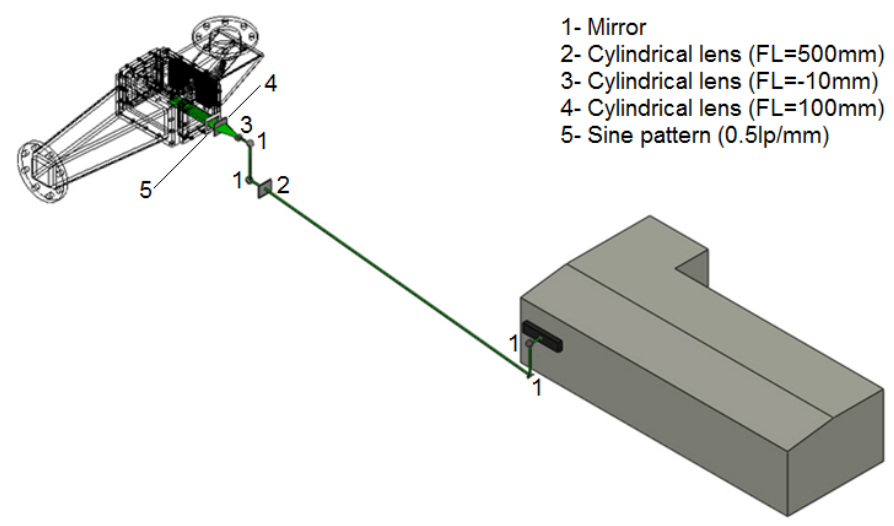

Figure 2: Illustration of the optical setup used in this study. The $\mathrm{Nd}$ :YAG laser is on the right (gray) and the testing section on the left (drawing lines).

flow is controlled and all the experiments have been done at a constant temperature set to $293 \mathrm{~K}$ to avoid evaporation.

\subsection{Illumination and acquisition systems}

To illuminate the spray with accuracy and precision, a pulsed Nd:YAG laser (Continuum - Surelite II) has been used. The beam has been steered toward the measurement section with three high-efficient $(99.5 \%$ at $532 \mathrm{~nm})$ laser-line mirrors. The laser-sheet used to illuminate sections of the spray has been generated by three cylindrical BK7 lenses. The first lens $(\mathrm{FL}=500 \mathrm{~mm})$ compresses the beam on the vertical dimension and is responsible of the thickness of the laser-sheet, the second one $(\mathrm{FL}=-10$ $\mathrm{mm}$ ) opens the beam on the horizontal direction to create a sheet. The third lens $(\mathrm{FL}=100 \mathrm{~mm})$ purpose is to concentrate the laser-sheet again and achieve parallel rays. The last element on the laser-sheet path is a sine-pattern filter that gives the illumination plane its structural behavior. The laser-sheet goes through this pattern and the spray is then illuminated by a spatial sinusoidal modulation with a period of $2 \mathrm{~mm}$.

Figure 2 presents an illustration of the optical arrangement used in this study. The characteristics of the illumination plane have been carefully specified as the objective was to get a laser-sheet thinner than the orifice of the injector, this in order to light all the spray on the central plane. The laser-sheet used in this study was $48 \mathrm{~mm}$ wide and $82 \mu \mathrm{m}$ thick (Full Width Half Maximum) on the axis of the spray.

The main organ of the acquisition system is a 12-bit CCD camera ( $P C O$ - Sensicam), the sensor has a resolution of $1376 \times 1040$ to ensure a relatively high pixel to millimeter ratio. The exposure time of the camera has been set to $1 \mu \mathrm{s}$ keeping the flare of the external environment as low as possible. Note that the laser power being relatively high compared to the ambient luminosity, the final gate of the image would be the laser pulse duration (about $5 \mathrm{~ns}$ ). 


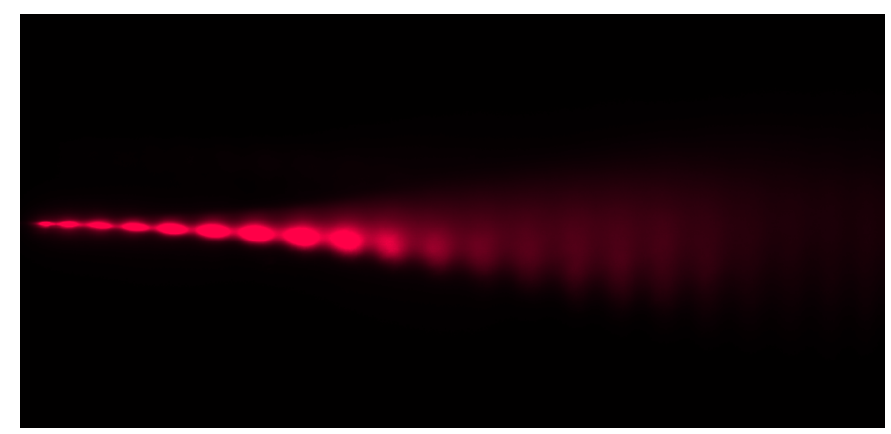

Figure 3: Example of a spray image illuminated by a sinusoidal modulation (false color). This is an average over 100 images of the LIF process. The spray is illuminated from the bottom.

The camera is equipped with a $60 \mathrm{~mm}$ focal length objective (Nikkor Lens). The collection angle has been set really low to reduce the effect of the multiple light scattering on the results. For this purpose, the diaphragm of the objective has been shut down to $f / \#=8$, being the final collection angle of the acquisition system as low as 23 mrad.

The same filters have been used to separate the two scattering processes: Mie (532 nm interference filter) and LIF (570 cut-off long-pass filter). Neutral density filters have been used to adjust the dynamic range of the acquisition system. For each phase, an amount of 100 images have been acquired to get statistically repetitive images, the images are acquired individually and the process of averaging is realized during the SPLI-2P image treatment. Figure 3 is a sample image of the spatial modulation illuminating a spray on its axis, the LIF signal is represented here with false colors.

The last element of the experimental apparatus set up to record the images of the spray via Structured Planar Laser Imaging is the electronic synchronization system. This equipment is necessary due to the really short time of the event, some milliseconds for the injection, some nanoseconds for the laser pulse. This purposely designed control system is based on a 16-bit micro-controller which communicates with a PC and generates the Transistortransistor logic (TTL) signals to trigger the injection, the flash-lamp and Q-switch of the laser, and the camera.

\subsection{Fluorescent properties of the dyed fuel}

The fuel used in this study is pure n-dodecane, it therefore does not absorb light and does not emit any fluorescent signal. A dye must be added to the fuel to record the corresponding fluorescent signal; sulforhodamine B has been chosen to dope the pure fuel because as many other dyes, it absorbs light at $532 \mathrm{~nm}$. Sulforhodamine B has been chosen for its high fluorescence efficiency which means that only a small quantity would be necessary to collect enough fluorescent signal.

Sulforhodamine B is not directly miscible with fuel and to achieve a complete and stable dilution of the dye, it has been dissolved into ethanol (1\% in volume) and the

\begin{tabular}{ccc}
\hline Properties & Values & Units \\
\hline Chemical formula & $\mathrm{C}_{27} \mathrm{H}_{30} \mathrm{~N}_{2} \mathrm{O}_{7} \mathrm{~S}_{2}$ & - \\
Molar weight & 558.666 & $\mathrm{~g} / \mathrm{mol}$ \\
Absorption (max. in ethanol) & 556 & $\mathrm{~nm}$ \\
Emission (max. in ethanol) & 575 & $\mathrm{~nm}$ \\
Molar absorptivity (532 nm) & $11.1 \times 10^{4}$ & $\mathrm{~m}^{2} / \mathrm{mol}$ \\
Fluorescence quantum yield & 0.68 & - \\
Fluorescence lifetime & 3.6 & $\mathrm{~ns}$ \\
\hline
\end{tabular}

Table 1: Fluorescence characteristics of sulforhodamine B added to n-dodecane.

mixture has been incorporate to the n-dodecane. Experiments to check both the fluorescent emission intensity and the non-existence of Morphology Dependent Resonance (MDR) have been carried out. Specific experiments revealed that the optimum quantity of sulfoRhodamine B to be added to the fuel was $12 \mathrm{mg} / \mathrm{L}\left(2.07 \times 10^{-5} \mathrm{~mol} / \mathrm{L}\right)$.

To better know the dye and its properties, several experiments have been performed such as absorption and emission spectra, fluorescent quantum yield and fluorescence lifetime. The results of the experiments concerning fluorescence characteristics of the dyes fuel are summarized in table 1 .

\subsection{Theoretical evaluation of the PDS parameters}

The Planar Droplet Sizing relies on collecting the scattered light and the fluorescent emission of an ensemble of droplets to make the ratio of both intensities which would provides a two-dimensional map of droplet diameter. This is true as long as the scattered light is proportional to the total surface of a droplet and the fluorescence to the droplet volume. It appeared that the scattered light intensity dependency with droplet surface was generally held, however the fluorescence intensity dependency with droplet volume was strongly affected by the dye content [14].

Both works ended concluding that the $d^{3}$ dependency was strongly affected by the dye content and that careful analysis was required prior to PDS experiments. This comes from the fact that if the absorption coefficient of the fluid is too high (for high dye concentration), the laser irradiance that reaches the other side of the droplet would be attenuated, thus affecting the intensity of the emission.

The methodology described by Domann and Hardalupas in ref.[14] has been applied to compute the fluorescence emission intensity dependency upon droplet diameter. The d-cube relationship has been calculated taking into account the absorption of light presented in table 1 within a fuel droplet. Figure 4 shows the results of the calculation; the best fitting is presented to evaluate the exponent for a droplet diameter ranging from 1 to 100 $\mu \mathrm{m}$.

The exponent obtained with the best match between simulation and fitting for the n-dodecane doped with sul- 


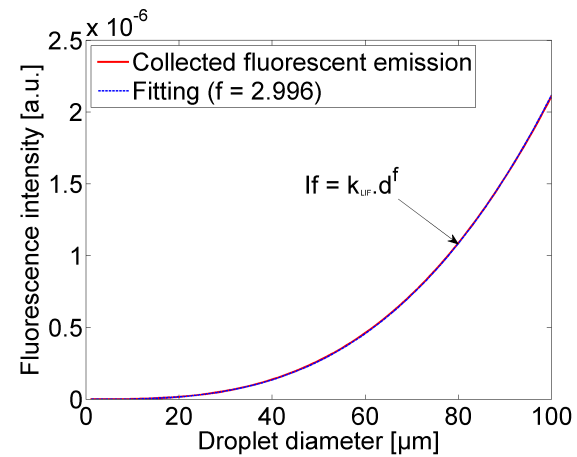

Figure 4: Fluorescence intensity as a function of droplet diameter for n-dodecane doped with sulforhodamine B. The curve has been fitted to measure the exponent on the droplet diameter.

forhodamine B is 2.996; this value is really close to 3 and would allow quantitative fluorescence measurements.

\section{Results validation and discussions}

This last main section presents the results of the experiments on isothermal Diesel sprays when the Structured Planar Laser Imaging methodology is applied. The technique has been used first to measure the droplet diameter using the PDS technique as introduced before. The intensity radiated by the laser-sheet toward the camera has been used then to recover the mixture fraction via the Beer-Lambert law. After validation of the results thanks to the extension of a $1-\mathrm{D}$ model into $2-\mathrm{D}$, the influence of experimental parameters on mixing is presented. This section also discusses the results and makes the link with the general knowledge concerning sprays.

\subsection{Spray sizing via Structured Planar Laser Imaging}

The Mie and LIF signals have been extracted via SPLI$2 \mathrm{P}$ and ratioed in order to obtain the droplet size distribution in the sprays injected in this work. The signal extracted from SPLI-2P represents the intensity of light scattered (or emitted, depending on the process) by the droplets present on the illumination plane.

The extinction of the laser-sheet in the spray has a strong impact on the measurements as the signal-to-noise ratio (SNR) decreases with the intensity of the illumination until zero when the signal gets too low. The extinction of light is therefore one of the principal limitations of the Structured Planar Laser Imaging technique as explained earlier. For this reason and with the sole intention of presenting accurate results, only half of the spray has been considered in this study. The axi-symmetrical aspect of modern Diesel sprays is a widespread concept [19], so as long as the axis of the spray is accurately determined, presenting half a spray seems to be a valid alternative.

Figure 5 presents a 2-D LIF/Mie ratio as well as two radial profiles at 25 and $45 \mathrm{~mm}$ downstream of the nozzle outlet. To extract the mixture fraction, the mean volume

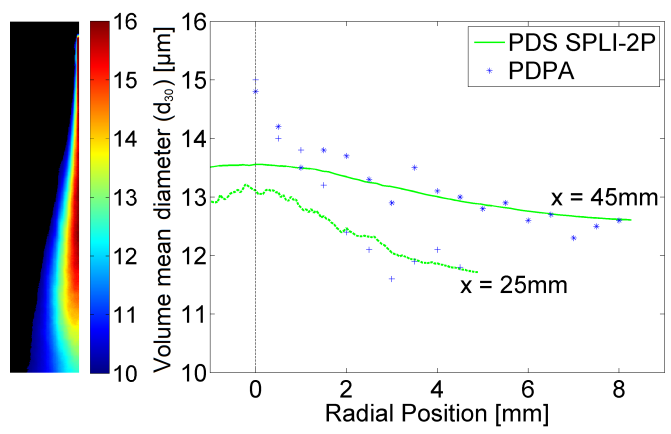

Figure 5: 2-D map of LIF/Mie ratio (SMD) in a Diesel spray calibrated through PDPA measurements of the volume mean diameter $d_{30}$ (left). Radial profiles of volume mean diameter via PDS when applying SPLI-2P method and PDPA at 25 and $45 \mathrm{~mm}$ (right).

diameter is required. Therefore, the LIF/Mie ratio has been adjusted with the mean volume diameter measured by the PDPA instrument at $45 \mathrm{~mm}$ on the edge of the spray (PDPA results for other locations are also plotted $[20])$.

The evolution of the droplet size with respect to axial and radial position for the LIF/Mie ratio, when Structured Planar Laser imaging is applied, agrees well with the results obtained through phase Doppler interferometry (PDPA) measurements. In addition to that, the results found throughout the literature [20] support the droplet size distribution presented in figure 5 . It has to be noted that the droplets near the axis of the spray measured via LIF/Mie ratio are smaller than the PDPA results. One explanation can be that the spray being denser at this location, the accuracy of the phase Doppler interferometer can be affected [21].

\subsection{Mixture fraction measurements and validation}

Prior to using the signals to compute the mixture fraction in the spray, another step is required. The SPLI-2P signals obtained do not account for the extinction of light between the illumination plane and the acquisition system. If this is not important for sizing as it is self-corrected by considering the ratio of Mie and LIF signals, it becomes crucial when a single signal is used. A three-dimensional correction technique has been implemented in this work to account for the extinction of light within the spray.

The solution consists in mapping the spray by imaging successive planes from the outside to inside of the spray until the illumination plane reaches the axis of the spray. This technique called discrete tomography [22] has been applied recently to evaluate the extinction coefficient in relatively dense sprays [23]. The procedure relies on the fact that the extinction between the illumination plane and the acquisition system follows the Beer-Lambert law.

In this experiment, the plane of illumination is shifted by a step size of a millimeter from outside the spray to the axis (being the axis the reference position). The first image is taken when the first droplets interact with the 
laser-sheet, at the outer boundary of the spray. At this position, the number density is still relatively low and thus the extinction toward the camera can be considered as negligible. Hence, the intensity radiated by the laser-sheet toward the camera is the signal processed by the SPLI-2P technique multiplied by the global efficiency of the system. From this first plane, the laser-sheet radiated intensity, the number density and the mixture fraction can be calculated for every integration area, i,e, a pixel. Then, by moving the laser-sheet toward the axis of the spray, the mixture fraction $Z$ (or fuel mass fraction for inert conditions) can be extracted for all the planes thanks to the following expression:

$$
Z=\frac{\rho_{f}}{\rho_{f}+\rho_{g}\left(\frac{6}{N \pi d_{30}^{3}}-1\right)}
$$

The mixture fraction is then a function of the droplet diameter, the number density $N$ as well as the densities of the fuel $\rho_{f}$ and the surrounding gas $\rho_{g}$. As it has been introduced earlier, an approximation has been made as the droplet diameter used in this equation is the volume mean diameter $d_{30}$ extracted through LIF/Mie ratio, which theoretically provides the $\operatorname{SMD}\left(d_{32}\right)$. This approximation is based on the assumption of a constant relationship between volume mean diameter and Sauter Mean Diameter. The analysis of this approximation confirmed that for the reference Diesel spray droplet diameter distribution, the maximum error made was as low as $1.5 \%$.

The mixing measurements have been confronted to a spray model to validate the experimental results. This model presented by Desantes et al. [12] and further improved by Pastor et al. [24] is based on gas-jet theory and conservation of momentum in the axial direction. The model also assumes a Gaussian radial distribution profile for both velocity and mass concentration expressed as follows:

$$
\frac{Z}{Z_{\text {axis }}}=\exp \left(-S c \alpha\left(\frac{r}{R}\right)^{2}\right)
$$

where $\alpha$ defines the limits of the Gaussian-like distribution profile. This parameter has been set to $\alpha=4.6$ in order to get a boundary equivalent to $1 \%$ value of the concentration on the centerline [19]. The ratio $r / R$ is the normalized radial position from the axis to the edge of the spray where the value of this ratio is 1 . The subscript "axis" represents the value on the centerline. $S c$ is the Schmidt number and represents the ratio of spray momentum diffusivity and mass diffusivity of the injected fluid such as:

$$
S c=\frac{\nu_{f}}{\kappa_{D}}
$$

In this expression, $\nu_{f}$ is the kinematic viscosity of the fluid which refers to the momentum diffusivity and $\kappa_{D}$ the mass diffusivity coefficient.

\begin{tabular}{ccc}
\hline Parameters & Values & Units \\
\hline Nozzle orifice diameter & 0.09 & $\mathrm{~mm}$ \\
Ambient density & 22.8 & $\mathrm{~kg} / \mathrm{m}$ \\
Fuel density & 720 & $\mathrm{~kg} / \mathrm{m}$ \\
Mass flow rate & 2.63 & $\mathrm{~g} / \mathrm{s}$ \\
Momentum flux & 1.54 & $\mathrm{~N}$ \\
Spreading angle & 17.3 & $\mathrm{o}$ \\
Schmidt number Sc & 0.8 & - \\
\hline
\end{tabular}

Table 2: Input parameters used in the spray model to simulate the experiments for the reference condition [17]: $p_{i n j}=150 \mathrm{MPa}$ and $\rho_{g}=22.8 \mathrm{~kg} / \mathrm{m}^{3}$.

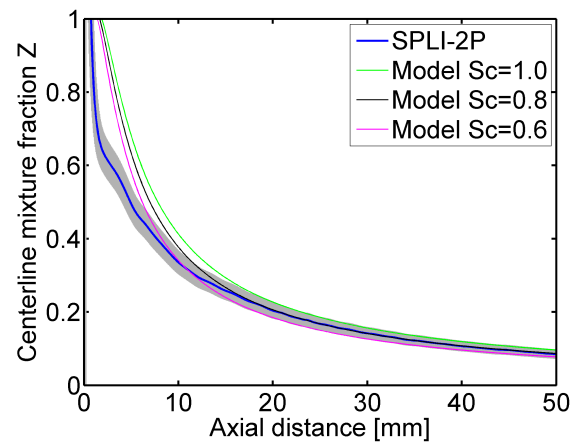

Figure 6: Experimental and computed mixture fraction on the spray centerline for the reference condition. The influence of Schmidt number on the centerline distribution is also shown.

Table 2 shows the parameters used as input to the model to simulate the experiments.

These parameters have been measured using the necessary equipment such as rate of injection meter, a purposelydesigned spray momentum test rig and macro-visualization of the injected spray to estimate the spreading angle [25].

Figure 6 presents the axial profile of the mixture fraction of a spray obtained with the SPLI-2P technique and confronted to the spray model. The Schmidt number has been varied from $S c=0.6$ to 1.0 to see the influence on mixing.

The results extracted via SPLI-2P are close to the predictions provided by the model when the Gaussian distribution defined in ref. [19] is assumed. The best agreement between measurements and predictions for the reference experimental condition has been found for $S c=0.8$. Despite the similarities concerning these results, it has to be noticed that in the region closer to the orifice, the experiments lie below the predictions. The model predictions fall within the uncertainties of the measurements (gray filled area) at roughly $10 \mathrm{~mm}$ from the exit of the nozzle. The uncertainties represented here are based on the results from previous experiments using fluorescent microspheres combined with the error on droplet size [8]. It has been observed that for short distances from the tip below 6 to $10 \mathrm{~mm}$ (depending on discharge density), the experimental error was higher due to a lack of accuracy when measuring 


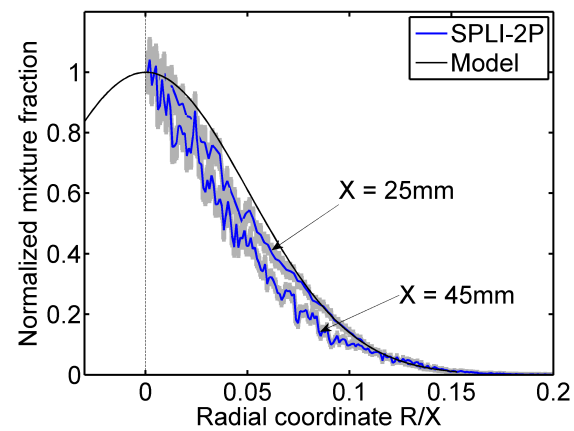

Figure 7: Normalized radial profiles of the mixture fraction at 25 and $45 \mathrm{~mm}$ from the tip for the reference condition. The predicted distribution assuming a Gaussian profile is also represented.

the extinction of light (due to a $1 \mathrm{~mm}$ step). The average uncertainty for the complete dataset has been estimated to be about $7 \%$ for distances longer than 6 to $10 \mathrm{~mm}$.

The validity of the results along the radial direction has been checked by plotting the normalized distributions at two distances from the tip (fig 7). The mixture fraction is normalized with respect to the centerline value at the corresponding position downstream $(\mathrm{X})$. The dimensionless radial coordinate $\mathrm{R} / \mathrm{X}$ is the spray radius divided by the axial position and allows direct comparison of the radial distribution profiles at different distances. The prediction provided by the model assuming the Gaussian profile described above (see eq. 13) is also plotted.

Such a representation of the results should show selfsimilarity of the radial distribution profiles for different distances downstream as long as the spreading angle is constant. The normalized radial distributions of the mixture fraction obtained by applying the Structured Planar Laser Imaging technique look similar for both axial distances (25 and $45 \mathrm{~mm}$ ). Both radial distributions resemble a Gaussian-like profile as widely suggested by the literature $[12,24]$, providing credits to these measurements. Nonetheless, by looking closer to the experimental radial distributions, it can be observed that both profiles appear slightly narrower than the predictions. This is particularly true at $45 \mathrm{~mm}$ downstream as the normalized mixture fraction seems to deviate more from the predicted Gaussian profile than the profile at $25 \mathrm{~mm}$. This has already been observed by Pastor et al. [18], they noticed that the fuel concentration profile (represented in dimensionless radial coordinate) was narrower for the furthest location measured from the orifice outlet.

As the measured radial distributions show slightly different behavior to the theoretical profile proposed in equation 13, this may suggest an inconsistency between liquid sprays and models based on gas-jet theory. The assumption of complete mixing of the injected fluid with the ambient gases may not hold for isothermal sprays. One simple explanation could be that the momentum transfer, which is supposed to be complete for gaseous jets, is not complete for two-phase flows like non-evaporative Diesel sprays. The next section presents an analysis of the influence of experimental conditions on mixing and provides some arguments to support the hypothesis of incomplete momentum transfer.

\subsection{Influence of experimental conditions on mixing}

The SPLI-2P technique has been applied to different experimental conditions in order to evaluate the influence of parameters such as injection pressure or ambient density on the development of sprays and global mixing process. These parameters have been varied widely; four injection pressures: 50, 100, 150 and $200 \mathrm{MPa}$ and three discharge densities: $7.6,22.8$ and $45.6 \mathrm{~kg} / \mathrm{m}^{3}$ have been selected. The injector energizing time has been set to $E T=2800 \mu \mathrm{s}$ as this time was long enough for the steady region of the spray to reach the end of the visualization window under all testing conditions.

The impact of ambient density in terms of mixing distribution is represented in figure 8 in which the resulting axial and radial profiles of the mixture fraction are plotted. The density of the ambient gases affects the behavior of the spray in terms of penetration rate [26] but also in terms of global mixing as both are ultimately related. Lower discharge density makes the spray to penetrate faster, but also to be narrower and therefore, to entrain less ambient gases in the stream. This affects the mixture fraction distribution because the sprays are then narrower and have a higher fuel concentration on the centerline. Under higher ambient density conditions, the contrary is observed, the sprays are wider, thus entraining the ambient gases at a higher rate. As a result, the mixture fraction on the centerline is lower and the Gaussian-like radial profile wider as seen in figure 8 .

This has an influence on the global mixing and further processes as the boundary for stoichiometric fuel-air ratio is observed to be at different locations depending on the discharge density. Taking into account that mixing is changing with time, it makes sense that the injection strategy is a key in the global internal combustion process of modern Direct-Injection engines $[2,5]$.

The injection pressure is another important parameter concerning spray development as it drives the injected mass flow rate [19]. The next two plots (fig. 9) present a confrontation of the resulting mixture fraction measured by the SPLI-2P technique when the injection pressure is varied.

The graphs show only slight differences between the mixture fraction distribution of the spray for injection pressures from 50 to $200 \mathrm{MPa}$ under a typical ambient density of $22.8 \mathrm{~kg} / \mathrm{m}^{3}$. Although the mixture fraction distributions on the centerline are really close (fig. 9a), it seems that higher injection pressure tends to make the radial profile a little wider (fig. 9b). It is difficult to appreciate small variations of the mixture fraction on the centerline in figure 9a, nevertheless, the differences are evident on 

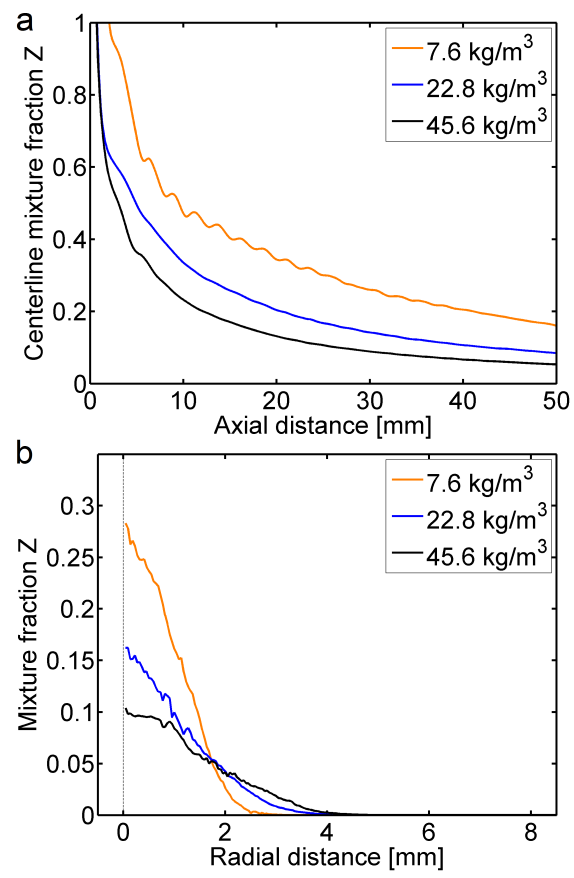

Figure 8: Measured mixture fraction of the isothermal spray on the centerline (a) and radially at $25 \mathrm{~mm}$ downstream (b). This graph shows the influence of ambient density on fuel-ambient mixing at constant injection pressure: $p_{i n j}=150 \mathrm{MPa}$.
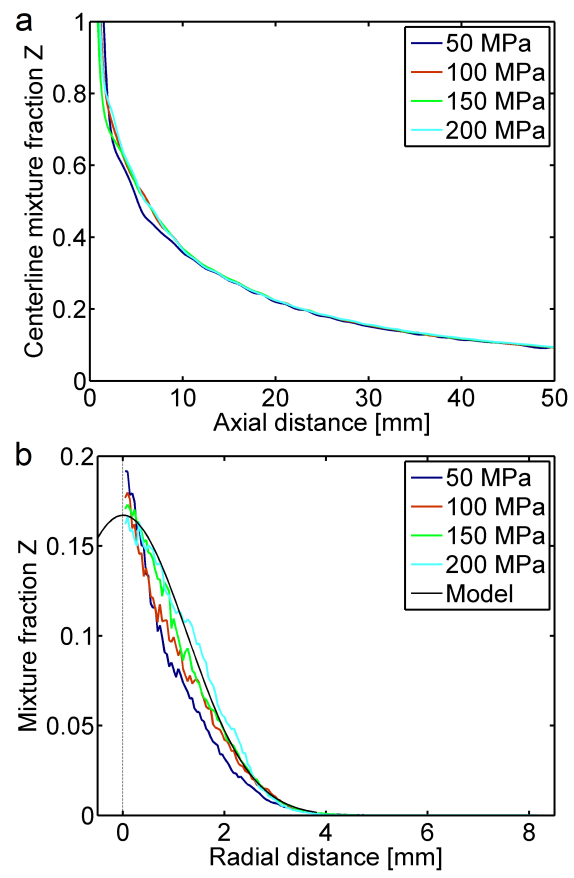

Figure 9: Measured mixture fraction on the centerline (a) and at 25 $\mathrm{mm}$ downstream (b) for different injection pressures. The predicted radial distribution is also plotted. $\left(\rho_{g}=22.8 \mathrm{~kg} / \mathrm{m}^{3}\right)$

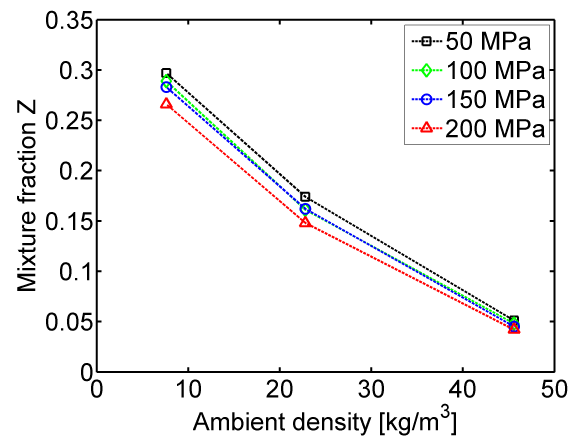

Figure 10: Graphical representation of the mixture fraction evolution as a function of ambient density for all injection pressures tested. The values are taken on the centerline at $25 \mathrm{~mm}$ from the tip.

the radial distributions. It can be noticed that the experimental radial distribution gets closer to the predictions for higher injection pressures; good agreement is found for $p_{i n j}=200 \mathrm{MPa}$. This falls in line with the hypothesis formulated earlier suggesting incomplete momentum transfer between the liquid spray and the ambient gases. Depending on the injection pressure, the entrainment rate of ambient gases into the spray stream is affected, thus at higher injection pressure, higher entrainment rate, better mixing. According to the results presented in figure 9b, a $200 \mathrm{MPa}$ injection pressure would produce sprays that mix completely with the ambient as suggested by the closeness to the predictions based upon the theory of gas-jets. Care must be taken with this as the predictions include a Schmidt number of $\mathrm{Sc}=0.8$, which means that the diffusivity ratio of momentum and mass for liquid sprays is different to that of gas-jets. Finally, it can be said about these results that even if the fuel is supplied at a higher mass flow, the entrainment rate of the ambient gases is balanced and hence, the mixture fraction remains similar.

In order to regroup the results concerning the mixture fraction distribution of an isothermal spray, the mixture fractions on the centerline at an axial distance of $25 \mathrm{~mm}$ have been graphically represented in figure 10. This graph is a compilation of the 12 different experimental conditions tested in this work.

The impact of the experimental conditions on global mixing of liquid sprays appears clearly in this figure. The effect of the ambient density has already been observed directly on the mixing distributions presented in figure 8 . As commented earlier, the ambient density affects the development of the spray, by increasing ambient density, the momentum flux of the injected spray "fights" against higher forces to penetrate in the ambient gases. This results in changing the ratio between axial and radial momentum diffusion components, the radial one becoming more important in this case. This means that the global mixing field of the spray is extended radially, thus entraining the ambient gases at a higher rate. The result of this is that the local mixture fraction gets lower when the entrainment rate of the ambient gases surrounding the spray 
increases. This effect can be summarized as the balance between spray momentum and ambient density as supported by the numerous works and models [26, 12] available to predict global mixing of sprays.

These works also pointed out the relationship between global mixing of the sprays and tip penetration as they are both closely related to momentum flux. The consequence of an increase of the injection pressure, while holding ambient density, makes the spray to penetrate faster in the chamber. However, as it has been commented earlier, this seems not to affect the mixing of sprays widely as the entrainment rate of the ambient gases into the spray stream is balanced. This makes the fuel concentration or mixture fraction not to change so much for those particular experimental conditions while mass flow rate was varied widely. Despite that, it can be seen on figure 10 that higher injection pressure produces lower mixture fraction at this location, which is consistent with the hypothesis of wider sprays formulated above.

This means that higher injection pressure enhances fuel - ambient mixing as commented earlier. Researches dedicated to spray sizing observed that an increment of the injection pressure led to sprays with droplets that are globally smaller [27]. This is the direct consequence of a higher velocity of the flow at the outlet of the orifice. The result is an intensification of the disintegration process (higher Weber number), ultimately responsible of the size of the droplets (if all the other parameters are held) [27]. The results show then that sprays with smaller droplets mix better with the ambient. At the same time, higher velocities in the orifice result in higher turbulence level inside but also outside the nozzle. This confirms what has been said above that sprays generated by high pressure drops tend to behave like gas-jets as a consequence of droplet size and turbulence intensity.

One last comment can be made on figure 10, the impact of injection pressure on the mixture fraction is lower for higher ambient densities (cf. $45.6 \mathrm{~kg} / \mathrm{m}^{3}$ in fig. 10). In fact, sprays injected under relatively high discharge density seem to entrain ambient better; as a consequence, injection pressure does not affect mixing so much as it is already quite efficient. Also, fuel - ambient mixing is related to spray tip penetration because the spray drags the surrounding gases in its stream. Naber and Siebers [26] conducted a series of tests to compare isothermal and evaporative spray development and also observed lower differences in penetration rate at high ambient densities. This can be understood as the fact that the development and mixing of sprays injected into high ambient density get closer to that of evaporative sprays (similar to gas-jets [28]).

\section{Conclusions}

A technique based on spatially modulated incident light and inspired by the recently developed Structured Laser Illumination Planar Imaging (SLIPI) has been developed to quantitatively measure the mixture fraction in isothermal DI Diesel sprays. An evaluation of the fluorescence properties demonstrated good agreement with square and cubic dependencies of the scattered and fluorescent emission with droplet diameter, respectively. A phase Doppler instrument has been used to get two-dimensional maps of droplet diameter via LIF/Mie ratio thanks to the signals extracted through the SPLI-2P technique. The comparison to a validated mixing model based upon gas-jet theory showed relatively good agreement, a Schmidt number of Sc $=0.8$ has been used to fit the experimental results better.

The results provided by the Structured Planar Laser Imaging technique showed that isothermal sprays present higher fuel concentration in the center than the predictions. The difference comes from that the momentum between liquid droplets and ambient gases is not completely transfered, increasing axial forces and lowering the radial ones, hence leading to narrower fuel distribution profiles. As expected, the impact of discharge density on fuel - ambient mixing has been observed to be quite important, leading to sprays that are more fuel rich at lower ambient density. On the other hand, it has been seen that the global mixing was enhanced for higher injection pressures, hence getting closer to the gas-jet-based predictions of the model. This is also related to the momentum transfer as sprays at higher injection pressures presents smaller droplets that are more likely to drag ambient gases in their stream. This means that care must be taken when using get-jet-based models to predict liquid spray development and mixing as gas-jets assume complete momentum transfer (Schmidt number equal to the unity).

\section{Acknowledgments}

This work has been financially supported by the national research project (ref.: TRA2006-15620-C02-02) provided by the Spanish Ministry of Science and Innovation. The authors would like to thank José Enrique del Rey (from CMT-Motores Térmicos, Universidad Politécnica de Valencia) for his collaboration during the experimental setup.

\section{References}

[1] S. Park, J. Cha, C. Lee, Effects of bioethanol-blended Diesel fuel on combustion and emission reduction characteristics in a direct-injection Diesel engine with exhaust gas recirculation (EGR), Energy \& Fuels 24 (2010) 3872-3883.

[2] S. Som, A. Ramirez, D. Longman, S. Aggarwal, Effect of nozzle orifice geometry on spray, combustion, and emission characteristics under Diesel engine conditions, Fuel 90 (3) (2010) $1267-1276$.

[3] W. Fimml, F. Chmela, G. Pirker, A. Wimmer, Influence of cavitation in the injection nozzle on combustion in Diesel engines, International Journal of Engine Research 11 (5) (2010) 375-390.

[4] C. Powell, A. Kastengren, Z. Liu, K. Fezzaa, The effects of Diesel injector needle motion on spray structure, Journal of Engineering for Gas Turbines and Power 133 (2011) 012802. 
[5] J. Kostas, D. Honnery, J. Soria, Time resolved measurements of the initial stages of fuel spray penetration, Fuel 88 (11) (2009) 2225-2237.

[6] S. H. Park, H. J. Kim, H. K. Suh, C. S. Lee, Experimental and numerical analysis of spray-atomization characteristics of biodiesel fuel in various fuel and ambient temperatures conditions, International Journal of Heat and Fluid Flow 30 (5) (2009) 960-970.

[7] C. Yeh, H. Kosaka, T. Kamimoto, A fluorescence/scattering imaging technique for instantaneous 2-D measurement of particle size distribution in a transient spray, 3rd International Congress on Optical Particle Sizing, Yokohama, Japan (1993) 355-361.

[8] J. Pastor, R. Payri, L. Araneo, J. Manin, Correction method for droplet sizing by laser-induced fluorescence in a controlled test situation, Optical Engineering 48 (2009) 013601.

[9] M. Paciaroni, M. Linne, Single-shot, two-dimensional ballistic imaging through scattering media, Applied optics 43 (26) (2004) 5100-5109.

[10] A. Ramrez, S. Som, S. Aggarwal, A. Kastengren, E. ElHannouny, D. Longman, C. Powell, Quantitative x-ray measurements of high-pressure fuel sprays from a production heavy duty diesel injector, Experiments in Fluids 47 (1) (2009) 119134.

[11] E. Kristensson, E. Berrocal, M. Richter, S. Pettersson, M. Aldèn, High-speed structured planar laser illumination for contrast improvement of two-phase flow images, Optics letters 33 (23) (2008) 2752-2754.

[12] J. Desantes, J. Lopez, J. Garcia, J. Pastor, Evaporative Diesel spray modeling, Atomization and Sprays 17 (3) (2007) 193-232.

[13] F. Payri, J. Pastor, R. Payri, J. Manin, Determination of the optical depth of a DI Diesel spray, Journal of Mechanical Science and Technology 25 (1) (2011) 209-219.

[14] R. Domann, Y. Hardalupas, Spatial distribution of fluorescence intensity within large droplets and its dependence on dye concentration, Applied Optics 40 (21) (2001) 3586-3597.

[15] M. Neil, R. Juskaitis, T. Wilson, Method of obtaining optical sectioning by using structured light in a conventional microscope, Optics Letters 22 (24) (1997) 1905-1907.

[16] T. Breuninger, K. Greger, E. Stelzer, Lateral modulation boosts image quality in single plane illumination fluorescence microscopy, Optics letters 32 (13) (2007) 1938-1940.

[17] CMT Motores Térmicos, Spray A Experimental Database, Universidad Politécnica de Valencia. URL http://www . cmt.upv .es/ECN . aspx

[18] J. Pastor, J. Lopez, J. Julia, J. Benajes, Planar laser-induced fluorescence fuel concentration measurements in isothermal diesel sprays, Optics Express 10 (7) (2002) 309-323.

[19] J. Desantes, R. Payri, J. Garcia, F. Salvador, A contribution to the understanding of isothermal Diesel spray dynamics, Fuel 86 (7-8) (2007) 1093-1101.

[20] R. Payri, B. Tormos, F. Salvador, L. Araneo, Spray droplet velocity characterization for convergent nozzles with three different diameters, Fuel 87 (15-16) (2008) 3176-3182.

[21] L. Araneo, V. Soare, R. Payri, J. Shakal, Setting up a PDPA system for measurements in a Diesel spray, Journal of Physics: Conference Series 45 (2006) 85.

[22] G. Herman, A. Kuba, Discrete tomography: Foundations, algorithms, and applications, Birkhauser.

[23] A. Berrocal, R. Wellander, E. Kristensson, Accounting for mulitple scattering signal attenuation and laser extinction using structured laser illumination planar imaging, ILASS-Europe 2010, Brno, Czech Republic.

[24] J. Pastor, J. Lopez, J. Garcia, J. Pastor, A 1-D model for the description of mixing-controlled inert diesel sprays, Fuel 87 (1314) (2008) 2871-2885.

[25] R. Payri, J. Garcia, J. Manin, M. Bardi, Fuel temperature influence on Diesel sprays in reacting conditions, ILASS-Americas 2011, Ventura, CA

[26] J. Naber, D. Siebers, Effects of gas density and vaporization on penetration and dispersion of diesel sprays, SAE Paper 960034
[27] C. Lee, S. Park, An experimental and numerical study on fuel atomization characteristics of high-pressure Diesel injection sprays, Fuel 81 (18) (2002) 2417-2423.

[28] L. Pickett, J. Manin, C. Genzale, D. Siebers, M. Musculus, C. Idicheria, Relationship between diesel fuel spray vapor penetration/dispersion and local fuel mixture fraction, SAE Paper 2011-01-0686.

Published as: Payri, Raúl, Gimeno, Jaime, Marti-Aldaravi, Pedro, Manin, J.. (2012). Fuel concentration in isothermal Diesel sprays through structured planar laser imaging measurements. International Journal of Heat and Fluid Flow, 34, 0, 98-106. 